\title{
A Coupled Elastoplastic Damage Model for Geomaterials
}

\author{
M.R. Salari and S. Saeb \\ RockSol Consulting Group, Inc., Boulder, Colorado \\ K.J. Willam \\ University of Colorado, Boulder \\ S.J. Patchet and R.C. Carrasco \\ Washington TRU Solutions, LLC, Carlsbad, New Mexico
}

\begin{abstract}
A triaxial constitutive model is developed for elastoplastic behavior of geomaterials, which accounts for tensile damage. The constitutive setting is formulated in the framework of continuum thermodynamics using internal variables. The interaction of elastic damage and plastic flow is examined with the help of very simple constitutive assumptions: (i) a Drucker-Prager yield function is used to define plastic loading of the material in combination with a non-associated flow rule to control inelastic dilatancy; (ii) elastic damage is assumed to be isotropic and is represented by a single scalar variable that evolves under expansive volumetric strain. Thereby, positive volumetric deformations couple the dissipation mechanisms of elastic damage and plastic flow which introduce degradation of the elastic stiffness as well as softening of the strength. The constitutive model is implemented in the finite element program ADINA to determine the response behavior of the combined damage-plasticity model under
\end{abstract}


displacement and mixed control. A number of load histories are examined to illustrate the performance of the material model in axial tension, compression, shear and confined compression. Thereby incipient failure is studied at the material level in the form of nonpositive properties of the tangential material tensor of elastoplastic damage and the corresponding localization tensor comparing non-associative with associative plasticity formulations.

\section{INTRODUCTION}

As underground excavations age, large-scale fractures may develop in the surrounding rock mass, becoming visible only after several years. The process of fracturing, however, starts immediately after excavation. Micro-cracks form during the excavation process in zones within the rock mass that are subjected to considerable redistribution of the initial stress state. As the micro-cracks grow, their distribution in the rock mass results in progressive deterioration of the strength and stiffness properties, and possible loss of stability of the surfaces of excavation. Micro-fracturing also has consequences on the hydrological performance of underground openings since it affects the porosity and permeability of the surrounding rock. Hence realistic geomaterial models not only of strength but also of stiffness and ductility degradation under triaxial conditions are a critical element to assess the long term performance of rock masses under excavations. For background information the reader is referred to seminal geomaterial models based on elastoplasticity, see Dafalias [1986], Desai [2001], Nova [1992], Borja and Tamagnini [1998], on hypoplasticity, see Darve [1991], Kolymbas [1991], and on continuum damage mechanics, see Mazars and Pijaudier-Cabot [1989], Dragon and Mroz [1979], Yazdani and Schreyer [1988], and Carol, Rizzi and Willam [2001], to name a few. 
Among recently proposed models for rock materials, the contributions by Shao et al. [1998], Nawrocki and Mroz [1999], Lee and Fenves [1998], and Hansen, Willam, K. and Carol [2001] are of direct interest to this paper.

Shao et al. [1998] developed a constitutive model to consider coupling between plastic deformations and damage induced by micro-cracks. The formulation was proposed to describe the salient features of hard clays, such as inelastic deformation, dilatancy, deterioration of elastic stiffness moduli and deformation-induced anisotropy. The plastic model was coupled with damage assuming that damage is induced by growth of microcracks related to dilatant volumetric deformation. Therefore, it was assumed that the energy due to tensile elastic and plastic volumetric strain is mainly responsible for the evolution of damage in tension as well as in compression.

In this paper, a constitutive model is developed for elastoplastic behavior of geomaterials, which accounts for tensile damage. The constitutive setting is formulated in the framework of continuum thermodynamics using internal variables. To illustrate the coupling of plasticity and elastic damage a Drucker-Prager yield function is used for plastic loading of the material and a non-associated flow rule is employed to control inelastic dilatancy. Damage is assumed to maintain isotropic elastic behavior and is represented by a scalar damage variable that evolves under volumetric expansion. The constitutive model is implemented in the finite element program ADINA [2001], and a number of load histories are examined to study the performance of the model. Although rate effects have been included in the geomaterial model, only the rate independent part of the constitutive formulation will be discussed in this article. Thereby it is understood that failure analysis of realistic initial boundary value problems must address well- 
posedness of the overall computational formulation, see Valanis and Peters [1996]. To set the stage, failure indicators are examined in the form of singularity diagnostics when the tangential elastoplastic damage tensor indicates loss of stability in the sense of nonpositive second order work and loss of uniqueness in the sense of non-positive, but real eigenvalues, see Willam [2002]. In addition, the corresponding acoustic material tensor is studied with regard to loss of positive wave speeds synonymous with localization and the formation of spatial discontinuities of the kinematic fields.

\section{THERMODYNAMICS FRAMEWORK}

In this section the coupled elastoplastic damage material model is formulated within the framework of continuum thermodynamics using internal variables. Assuming an isothermal process, the Helmholtz free energy is considered to depend on three state variables:

$$
\psi=\psi\left(\boldsymbol{\varepsilon}^{e}, \kappa, D\right)
$$

where $\boldsymbol{\varepsilon}^{e}$ denotes the elastic strain tensor and $\kappa$ and $D$ the scalar-valued internal variables of plasticity and damage. Assuming that the Helmholtz free energy may be additively decomposed into elastic and plastic components:

$$
\psi=\psi^{e}\left(\boldsymbol{\varepsilon}^{e}, D\right)+\psi^{p}(\kappa, D)
$$

which are however both dissipative because of elastic damage which couples both energy contributions. To ensure that the second principle of thermodynamics is satisfied, the local Clausius-Duhem's inequality requires that the reduced dissipation inequality holds:

$$
\boldsymbol{\sigma}: \dot{\boldsymbol{\varepsilon}}-\dot{\psi} \geq 0
$$


Evaluation of this inequality involves the time derivative of the Helmholtz free energy:

$$
\dot{\psi}=\frac{\partial \psi^{e}}{\partial \boldsymbol{\varepsilon}^{e}} \dot{\boldsymbol{\varepsilon}}^{e}+\frac{\partial \psi^{e}}{\partial D} \dot{D}+\frac{\partial \psi^{p}}{\partial \kappa} \dot{\kappa}+\frac{\partial \psi^{p}}{\partial D} \dot{D}
$$

Substitution into the reduced dissipation inequality results in:

$$
\left(\boldsymbol{\sigma}-\frac{\partial \psi^{e}}{\partial \boldsymbol{\varepsilon}^{e}}\right) \dot{\boldsymbol{\varepsilon}}+\frac{\partial \psi^{e}}{\partial \boldsymbol{\varepsilon}^{e}} \dot{\boldsymbol{\varepsilon}}^{p}-\frac{\partial \psi^{p}}{\partial \kappa} \dot{\kappa}-\frac{\partial \psi}{\partial D} \dot{D} \geq 0
$$

where the additive decomposition into elastic and plastic strain contributions has been used with $\dot{\boldsymbol{\varepsilon}}^{p}$ denoting the plastic strain tensor. Since the inequality (5) must hold for any value of $\dot{\boldsymbol{\varepsilon}}, \dot{\boldsymbol{\varepsilon}}^{p}, \dot{\boldsymbol{\kappa}}, \dot{D}$ the Coleman relations yield the constitutive expressions:

$$
\boldsymbol{\sigma}=\frac{\partial \psi^{e}}{\partial \boldsymbol{\varepsilon}^{e}}
$$

and the thermodynamic conjugate forces for plasticity and damage:

$$
\begin{gathered}
K=-\frac{\partial \psi^{p}}{\partial \kappa} \\
Y=-\frac{\partial \psi}{\partial D}
\end{gathered}
$$

Assuming that the plastic and damage potential functions $g^{p}$ and $g^{d}$ are functions of the thermodynamic forces and the scalar damage variable we have:

$$
\begin{gathered}
g^{p}=g^{p}(\boldsymbol{\sigma}, K ; D) \\
g^{d}=g^{d}(Y ; D)
\end{gathered}
$$

where $g^{p}$ is also a function of stress in order to render the tensorial format for the rate of plastic strain. Appropriate evolution laws characterize the rate of change of the internal variables as: 


$$
\begin{gathered}
\dot{\boldsymbol{\varepsilon}}^{p}=\dot{\lambda} \frac{\partial g^{p}}{\partial \boldsymbol{\sigma}} \\
\dot{\kappa}=\dot{\lambda} \frac{\partial g^{p}}{\partial K} \\
\dot{D}=\dot{\mu} \frac{\partial g^{d}}{\partial Y}
\end{gathered}
$$

Hereby $\dot{\lambda} \geq 0$ and $\dot{\mu} \geq 0$ denote the plastic and damage multipliers, respectively. Considering $f^{p}=f^{p}(\boldsymbol{\sigma}, K, D)$ for plastic loading and $f^{d}=f^{d}(Y, D)$ for damage initiation, respectively, the two consistency conditions,

$$
\begin{gathered}
\dot{f}^{p}=\frac{\partial f^{p}}{\partial \boldsymbol{\sigma}}: \dot{\boldsymbol{\sigma}}+\frac{\partial f^{p}}{\partial K} \dot{K}+\frac{\partial f^{p}}{\partial D} \dot{D}=0 \\
\dot{f}^{d}=\frac{\partial f^{d}}{\partial Y} \dot{Y}+\frac{\partial f^{d}}{\partial D} \dot{D}=0
\end{gathered}
$$

enforce persistent elastoplastic and damage behavior in the form of two coupled equations which determine the relative magnitude of plastic vs. damage dissipation.

\section{PLASTICITY FORMULATION}

For the sake of simplicity the pressure-sensitive Drucker-Prager model is used to describe the plastic response behavior, where the plastic loading function,

$$
f^{p}\left(\boldsymbol{\sigma}, \bar{e}^{p}, D\right)=\alpha I_{1}+\sqrt{J_{2}}-(1-D) k
$$

has been modified to consider the effect of damage in the cohesive resistance. Hereby, $I_{1}=\sigma_{k k}$ denotes the first invariant of the 'nominal' stress tensor $\boldsymbol{\sigma}, J_{2}=1 / 2 s_{i j} s_{i j}$ the second invariant of the deviatoric stress tensor, $\bar{e}^{p}$ the effective deviatoric plastic strain, and $D$ the scalar-valued damage parameter. The two Drucker-Prager parameters $\alpha$ and 
$k$ are a measure of internal material friction and cohesion, respectively, which may be expressed in terms of the uniaxial tensile and compressive strength values as:

$$
\alpha=\frac{1}{\sqrt{3}} \frac{f_{c}-f_{t}}{f_{c}+f_{t}} \quad \text { and } \quad k=\frac{2}{\sqrt{3}} \frac{f_{c} f_{t}}{f_{c}+f_{t}}
$$

Traditionally, damage is introduced in the plastic loading function in the form of effective rather than nominal stress. This is entirely equivalent to the effect of damage on the cohesive strength in Eq. (15), when the loading function is divided by $(1-D)$.

Assuming that plastic hardening depends only on the equivalent deviatoric plastic strain $\bar{e}^{p}$ rather then the total plastic strain, the material parameters $\alpha$ and $k$ are expressed in terms of the exponential functions proposed by Shao et al. [1998]:

$$
\begin{gathered}
\alpha=\alpha_{m}-\left(\alpha_{m}-\alpha_{0}\right) e^{-b_{1} \bar{e}^{p}} \\
k=k_{m}-\left(k_{m}-k_{0}\right) e^{-b_{2} \bar{e}^{p}}
\end{gathered}
$$

where $\alpha_{0}, \alpha_{m}, k_{0}$, and $k_{m}$ are the initial and maximum values of the frictional and cohesive parameters $\alpha$ and $k$, respectively. The equivalent deviatoric plastic strain $\bar{e}^{p}$ is defined in terms of the Odquist parameter which is traditionally used in $J_{2}$-plasticity to express plastic dissipation in terms of von Mises stress and the equivalent palstic strain rate:

$$
\begin{gathered}
\bar{e}^{p}=\int_{0}^{\bar{e}^{p}} d \bar{e}^{p} \\
d \bar{e}^{p}=\sqrt{\frac{2}{3} d \mathbf{e}^{p}: d \mathbf{e}^{p}}
\end{gathered}
$$


Here $d \mathbf{e}^{p}$ denotes the rate of deviatoric plastic strain. Note, the volumetric part of the plastic strain rate does not affect plastic hardening, instead it will be used below to mobilize damage and henceforth softening rather than hardening.

To determine the direction of plastic strain rate, the following modification of the Drucker-Prager loading function is used as plastic potential:

$$
g^{p}\left(\boldsymbol{\sigma}, \bar{e}^{p}\right)=\beta I_{1}+\sqrt{J_{2}}
$$

Here the dilatation parameter $\beta$ is used to control inelastic volume expansion. Adopting a non-associated flow rule:

$$
\dot{\boldsymbol{\varepsilon}}^{p}=\dot{\lambda} \frac{\partial g}{\partial \boldsymbol{\sigma}}
$$

the rate of change of mean plastic strain $\varepsilon_{m}^{p}$ and deviatoric plastic strain $\mathbf{e}^{p}$ is defined by:

$$
\begin{gathered}
\dot{\varepsilon}_{m}^{p}=\dot{\lambda} \beta \\
\dot{\mathbf{e}}^{p}=\dot{\lambda} \frac{\mathbf{s}}{2 \sqrt{J_{2}}}
\end{gathered}
$$

Plastic consistency assures that the stress state remains on the yield surface during persistent plastic loading, i.e.

$$
\dot{f}^{p}=\frac{\partial f^{p}}{\partial \boldsymbol{\sigma}}: \dot{\boldsymbol{\sigma}}+\frac{\partial f^{p}}{\partial \bar{e}^{p}} \dot{\bar{e}}^{p}+\frac{\partial f^{p}}{\partial D} \dot{D}=0
$$


The rate of change of the state of stress in (6) involves two terms in the presence of damage:

$$
\dot{\boldsymbol{\sigma}}=\mathbf{E}^{d}: \dot{\boldsymbol{\varepsilon}}^{e}+\dot{\mathbf{E}}^{d}: \boldsymbol{\varepsilon}^{e}
$$

where $\mathbf{E}^{d}$ denotes the secant tensor of elastic damage and $\boldsymbol{\varepsilon}^{e}$ the elastic strain tensor. In the case of single scalar format of isotropic damage the degraded elastic stiffness tensor reads,

$$
\mathbf{E}^{d}=(1-D) \mathbf{E}^{0}
$$

Differentiating and substituting the results into (26) one obtains:

$$
\dot{\boldsymbol{\sigma}}=\mathbf{E}^{d}:\left(\dot{\boldsymbol{\varepsilon}}-\dot{\lambda} \frac{\partial g^{p}}{\partial \boldsymbol{\sigma}}\right)-\dot{D} \mathbf{E}^{0}: \boldsymbol{\varepsilon}^{e}
$$

Introducing the deviatoric plastic strain rate $\dot{e}_{i j}^{p}$ (24) into (20), the equivalent deviatoric plastic strain rate $\dot{\bar{e}}^{p}$ reduces to:

$$
\dot{\bar{e}}^{p}=\frac{\dot{\lambda}}{\sqrt{3}}
$$

Finally, the plastic consistency condition may be expressed in terms of the unknown plastic and damage multipliers and the prescribed strain rate as:

$$
\left(\frac{\partial f^{p}}{\partial \boldsymbol{\sigma}}: \mathbf{E}^{d}: \frac{\partial g^{p}}{\partial \boldsymbol{\sigma}}-\frac{\partial f^{p}}{\partial \bar{e}^{p}} \frac{1}{\sqrt{3}}\right) \dot{\lambda}+\left(\frac{\partial f^{p}}{\partial \boldsymbol{\sigma}}: \mathbf{E}^{0}: \boldsymbol{\varepsilon}^{e}-\frac{\partial f^{p}}{\partial D}\right) \dot{D}=\frac{\partial f^{p}}{\partial \boldsymbol{\sigma}}: \mathbf{E}^{d}: \dot{\boldsymbol{\varepsilon}}
$$


Under strain control, i.e. for a given rate of strain the internal rate of change of the plastic and damage $\dot{\lambda}$ and $\dot{D}$ determine together with their thermodynamic forces the magnitude of plastic and damage dissipation, respectively.

\section{DAMAGE FORMULATION}

In rock and concrete materials, damage is induced by micro-cracking which manifests itself at the macroscopic level in the form of expansive volumetric strain. Consequently, the tensile contribution of volumetric strain energy is mainly responsible for the evolution of damage. Following the proposal by Shao et al [1998], the volumetric part of the thermodynamic damage force drives the evolution of damage in the form:

$$
Y_{v}=\frac{1}{2} K^{0}\left\langle\varepsilon_{v}^{e}\right\rangle^{2}+c \int_{0}^{\varepsilon_{v}^{p}}\left|\sigma_{m}\right|\left\langle d \varepsilon_{v}^{p}\right\rangle
$$

where

$$
\begin{array}{ll}
c=c_{c} & \text { for } \varepsilon_{v}^{e}<0 \\
c=c_{t} & \text { for } \varepsilon_{v}^{e}>0
\end{array}
$$

and where $K^{0}$ is the undamaged bulk modulus. The plastic damage parameter $c$ controls the amount of coupling which the dilatant volumetric plastic energy dissipation contributes to the thermodynamic damage force. The bracket $\langle x\rangle$ around the argument denotes the McAuley discontinuity function, i.e. $\langle x\rangle=x$ when $x>0$ and $\langle x\rangle=0$ otherwise. Since damage in tension is very different from that in compression, different values $c_{t}$ and $c_{c}$ are introduced depending on the sign of the volumetric elastic strain. 
The energy-based damage function defines damage initiation in the form of the loading function:

$$
f^{d}\left(Y_{v}, D\right)=Y_{v}-r(D)
$$

where the volumetric thermodynamic force represents the energy demand and where the energy resistance function $r(D)$ is to be a power function, see Carol et al [2001]:

$$
r(D)=r_{\mathrm{o}}(1-D)^{p-1}
$$

Here $r_{0}$ designates the modulus of resilience, i.e. the volumetric strain energy at peak stress in uniaxial tension as shown in Figure 1, while the exponent $p$ represents the ratio between the modulus of resilience and the modulus of toughness:

$$
p=\frac{r_{\mathrm{o}}}{g_{f}}
$$

Figure 1 shows the uniaxial tension test and the physical meaning of $r_{\mathrm{o}}$ and $g_{f}$, whereby the latter may be interpreted as volumetric fracture energy release per unit volume.

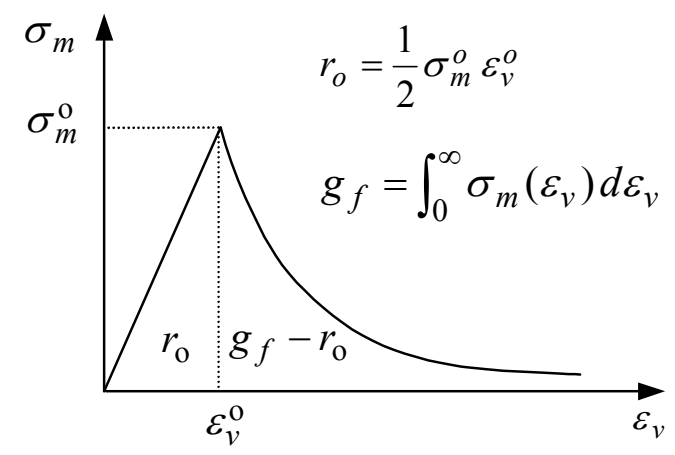

Figure 1. Uniaxial stress-strain curve 
Damage consistency assures that the stress state satisfies the damage function under persistent damage. Taking partial derivatives of the damage function with regard to the independent variables the consistency condition involves,

$$
\dot{f}^{d}=\frac{\partial f^{d}}{\partial Y_{v}} \dot{Y}_{v}+\frac{\partial f^{d}}{\partial D} \dot{D}=0
$$

Considering the definition of the volumetric thermodynamic force in (31), its rate of change reads:

$$
\dot{Y}_{v}=K^{0}\left\langle\varepsilon_{v}^{e}\right\rangle \dot{\varepsilon}_{v}^{e}+c\left|\sigma_{m}\right|\left\langle\dot{\varepsilon}_{v}^{p}\right\rangle
$$

Decomposing the volumetric strain into elastic and plastic parts and substituting the volumetric plastic strain rate into (36) results in:

$$
\dot{Y}_{v}=K^{0}\left\langle\varepsilon_{v}^{e}\right\rangle \mathbf{I}: \dot{\boldsymbol{\varepsilon}}-3 \dot{\lambda}\left(K^{0}\left\langle\varepsilon_{v}^{e}\right\rangle \frac{\partial g^{p}}{\partial I_{1}}-c\left|\sigma_{m}\right|\left\langle\frac{\partial g^{p}}{\partial I_{1}}\right\rangle\right)
$$

where $\mathbf{I}$ is the $2^{\text {nd }}$ order identity tensor and $\dot{\boldsymbol{\varepsilon}}$ is the rate of strain tensor. Finally, substituting (37) into (35) results in the second consistency condition analogous to (29):

$$
-3 R_{d}\left(K^{0}\left\langle\varepsilon_{v}^{e}\right\rangle \frac{\partial g^{p}}{\partial I_{1}}-c\left|\sigma_{m}\right|\left\langle\frac{\partial g^{p}}{\partial I_{1}}\right\rangle\right) \dot{\lambda}+\dot{D}=-R_{d} K^{0}\left\langle\varepsilon_{v}^{e}\right\rangle \mathbf{I}: \dot{\boldsymbol{\varepsilon}}
$$

where

$$
R_{d}=\frac{\frac{\partial f^{d}}{\partial Y_{v}}}{\frac{\partial f^{d}}{\partial D}} \quad \text { with } \quad R_{d}<0
$$


Considering (30) and (38) these are to simultaneous equations which determine the magnitude of the plastic and damage multipliers. Formally, the two equations for the unknown multipliers may be written in matrix notation as

$$
\left[\begin{array}{ll}
A_{11} & A_{12} \\
A_{21} & A_{22}
\end{array}\right]\left[\begin{array}{c}
\dot{\lambda} \\
\dot{D}
\end{array}\right]=\left[\begin{array}{c}
B_{1} \\
B_{2}
\end{array}\right] \text { with }\left[\begin{array}{c}
\dot{\lambda} \\
\dot{D}
\end{array}\right]=\frac{1}{A_{11} A_{22}-A_{12} A_{21}}\left[\begin{array}{cc}
A_{22} & -A_{12} \\
-A_{21} & A_{11}
\end{array}\right]\left[\begin{array}{c}
B_{1} \\
B_{2}
\end{array}\right]
$$

Thereby it is understood hat the two multipliers can be only positive or zero depending on the loading conditions in analogy to multi-surface plasticity models. In other terms, if one of the two multipliers turns out to be negative then the dissipation of this process is arrested while the other is active and vice versa. In summary, the loading conditions require that the coefficient matrix is positive, whereby $A_{11}>0$ and $A_{22}>0$ for plastic loading separately from damage, and where $A_{11} A_{22}>A_{12} A_{21}$ must hold for combined loading together with,

(a) $\dot{D}>0$ if $\quad A_{11} B_{2}-A_{21} B_{1}>0$

(b) $\dot{\lambda}>0$ if $A_{22} B_{1}-A_{12} B_{2}>0$

Hereby the individual coefficients in the matrix relations are defined in (30) and (38). Solution of the two simultaneous equations leads to an explicit form of the plastic multiplier $\dot{\lambda}$ in terms of the prescribed strain rate $\dot{\varepsilon}$ :

$$
\dot{\lambda}=\frac{\frac{\partial f^{p}}{\partial \boldsymbol{\sigma}}: \mathbf{E}^{d}: \dot{\boldsymbol{\varepsilon}}+R_{d}\left(\frac{\partial f^{p}}{\partial \boldsymbol{\sigma}}: \mathbf{E}^{0}: \boldsymbol{\varepsilon}^{e}-\frac{\partial f^{p}}{\partial D}\right) K^{0}\left\langle\varepsilon_{v}^{e}\right\rangle \mathbf{I}: \dot{\boldsymbol{\varepsilon}}}{\frac{\partial f^{p}}{\partial \boldsymbol{\sigma}}: \mathbf{E}^{d}: \frac{\partial g^{p}}{\partial \boldsymbol{\sigma}}-\frac{\partial f^{p}}{\partial \bar{e}^{p}} \frac{1}{\sqrt{3}}+3 R_{d}\left(\frac{\partial f^{p}}{\partial \boldsymbol{\sigma}}: \mathbf{E}^{0}: \boldsymbol{\varepsilon}^{e}-\frac{\partial f^{p}}{\partial D}\right)\left(K^{0}\left\langle\varepsilon_{v}^{e}\right\rangle \frac{\partial g^{p}}{\partial I_{1}}-c\left|\sigma_{m}\right\rangle\left\langle\frac{\partial g^{p}}{\partial I_{1}}\right\rangle\right)}
$$


Note strain control of the plastic multiplier introduces an additional term in the numerator as compared to classical plasticity and in the denominator which is augmented by an additional coupling term due to damage.

Vice versa, the rate of change of the damage variable is according to (38)

$$
\dot{D}=-R_{d} K^{0}\left\langle\varepsilon_{v}^{e}\right\rangle \mathbf{I}: \dot{\boldsymbol{\varepsilon}}+3 R_{d}\left(K^{0}\left\langle\varepsilon_{v}^{e}\right\rangle \frac{\partial g^{p}}{\partial I_{1}}-c\left|\sigma_{m}\right|\left\langle\frac{\partial g^{p}}{\partial I_{1}}\right\rangle\right) \dot{\lambda}
$$

Note the first term on the rhs is the traditional damage evolution expression under strain control while the second term on the rhs arises due to coupling between the damage and the plastic dissipation process.

\section{ELASTOPLASTIC DAMAGE TANGENT OPERATOR}

The elastoplastic damage tangent operator is obtained by substituting the rates of the internal variables $\dot{D}$ and $\dot{\lambda}$ from (38) and (41) into the constitutive rate equation (28):

$$
\dot{\boldsymbol{\sigma}}=\mathbf{E}^{e p d}: \dot{\boldsymbol{\varepsilon}}
$$

where $\mathbf{E}^{\text {epd }}$ designates the tangential elastoplastic damage operator:

$$
\begin{aligned}
& \mathbf{E}^{e p d}=\mathbf{E}^{d}+R_{d} K^{0}\left\langle\varepsilon_{v}^{e}\right\rangle \mathbf{I} \otimes\left(\mathbf{E}^{0}: \boldsymbol{\varepsilon}^{e}\right)_{-} \\
& \frac{\left(\mathbf{E}^{d}: \frac{\partial f^{p}}{\partial \boldsymbol{\sigma}}+R_{d}\left(\frac{\partial f^{p}}{\partial \boldsymbol{\sigma}}: \mathbf{E}^{0}: \boldsymbol{\varepsilon}^{e}-\frac{\partial f^{p}}{\partial D}\right) K^{0}\left\langle\varepsilon_{v}^{e}\right\rangle \mathbf{I}\right) \otimes\left(\mathbf{E}^{d}: \frac{\partial g^{p}}{\partial \boldsymbol{\sigma}}+3 R_{d}\left(K^{0}\left\langle\varepsilon_{v}^{e}\right\rangle \frac{\partial g^{p}}{\partial I_{1}}-c\left|\sigma_{m}\right\rangle\left\langle\frac{\partial g^{p}}{\partial I_{1}}\right\rangle\right) \mathbf{E}^{0}: \boldsymbol{\varepsilon}^{e}\right)}{\left.\frac{\partial f^{p}}{\partial \boldsymbol{\sigma}}: \mathbf{E}^{d}: \frac{\partial g^{p}}{\partial \boldsymbol{\sigma}}-\frac{\partial f^{p}}{\partial \bar{e}^{p}} \frac{1}{\sqrt{3}}+3 R_{d}\left(\frac{\partial f^{p}}{\partial \boldsymbol{\sigma}}: \mathbf{E}^{0}: \boldsymbol{\varepsilon}^{e}-\frac{\partial f^{p}}{\partial D}\right)\left(K^{0}\left\langle\varepsilon_{v}^{e}\right\rangle \frac{\partial g^{p}}{\partial I_{1}}-c \mid \sigma_{m}\right)\left\langle\frac{\partial g^{p}}{\partial I_{1}}\right\rangle\right)}
\end{aligned}
$$


In the special case when no damage is mobilized, (44) reduces to the traditional elastoplastic tangent operator:

$$
\mathbf{E}^{e p}=\mathbf{E}^{0}-\frac{\left(\mathbf{E}^{0}: \frac{\partial f^{p}}{\partial \boldsymbol{\sigma}}\right) \otimes\left(\mathbf{E}^{0}: \frac{\partial g^{p}}{\partial \boldsymbol{\sigma}}\right)}{\frac{\partial f^{p}}{\partial \boldsymbol{\sigma}}: \mathbf{E}^{0}: \frac{\partial g^{p}}{\partial \boldsymbol{\sigma}}-\frac{\partial f^{p}}{\partial \bar{e}^{p}} \frac{1}{\sqrt{3}}}
$$

In the absence of plasticity, the elastic damage tangent operator reduces (44) to:

$$
\mathbf{E}^{e d}=\mathbf{E}^{d}+R_{d} K^{0}\left\langle\varepsilon_{v}^{e}\right\rangle \mathbf{I} \otimes\left(\mathbf{E}^{0}: \boldsymbol{\varepsilon}^{e}\right)
$$

In general, the elastoplastic damage operator of (44) is non-symmetric, as is the case of elastoplasticity and elastic damage. Loss of plastic symmetry is due to the nonassociated flow rule, while loss of damage symmetry results from the fact that only the volumetric part of the strain tensor contributes to the damage formulation.

\section{STRESS INTEGRATION}

Numerical implementation of the model requires integrating the rate form of the constitutive relations in the finite time step ${ }^{n+1} \Delta t={ }^{n+1} t-{ }^{n} t$. Given the material response at time ${ }^{n} t$ and a finite strain increment ${ }^{n+1} \Delta \boldsymbol{\varepsilon}$, the objective is to determine the unknown external and internal state variables ${ }^{n+1} \boldsymbol{\sigma},{ }^{n+1} \boldsymbol{\varepsilon},{ }^{n+1} \boldsymbol{\varepsilon}^{p}$ and ${ }^{n+1} D$ at time ${ }^{n+1} t$. To this end, the fully implicit Euler backward integration method is used where the stress tensor at time ${ }^{n+1} t$ is updated:

$$
{ }^{n+1} \boldsymbol{\sigma}={ }^{n} \boldsymbol{\sigma}+{ }^{n+1} \Delta \boldsymbol{\sigma}
$$


by the finite stress increment ${ }^{n+1} \Delta \boldsymbol{\sigma}$ :

$$
{ }^{n+1} \Delta \boldsymbol{\sigma}={ }^{n} \mathbf{E}^{d}:{ }^{n+1} \Delta \boldsymbol{\varepsilon}^{e}+{ }^{n+1} \Delta \mathbf{E}^{d}:{ }^{n+1} \boldsymbol{\varepsilon}^{e}
$$

In scalar damage, progressive damage of the elastic stiffness involves:

$$
{ }^{n+1} \mathbf{E}^{d}={ }^{n} \mathbf{E}^{d}+{ }^{n+1} \Delta \mathbf{E}^{d}
$$

The reduction of stiffness is controlled by the change of the scalar damage variable,

$$
{ }^{n+1} \Delta \mathbf{E}^{d}=-{ }^{n+1} \Delta D \mathbf{E}^{0}
$$

Substituting (50) and using the strain decomposition results in the traditional elastic predictor-plastic corrector format of computational plasticity, see Simo and Hughes [1998],

$$
{ }^{n+1} \boldsymbol{\sigma}={ }^{n+1} \boldsymbol{\sigma}^{\text {trial }}\left(\frac{1-{ }^{n+1} D}{1-{ }^{n} D}\right)-{ }^{n+1} \mathbf{E}^{d}:{ }^{n+1} \Delta \boldsymbol{\varepsilon}^{p}
$$

Here the trial stress ${ }^{n+1} \boldsymbol{\sigma}^{\text {trial }}$ is defined explicitly as:

$$
{ }^{n+1} \boldsymbol{\sigma}^{\text {trial }}={ }^{n} \boldsymbol{\sigma}+{ }^{n} \mathbf{E}^{d}:{ }^{n+1} \Delta \boldsymbol{\varepsilon}
$$

A graphical representation of (51) for uniaxial loading is shown in Figure 2 when the trial stress ${ }^{n+1} \boldsymbol{\sigma}^{\text {trial }}$ is explicitly determined in terms of the damaged stiffness ${ }^{n} \mathbf{E}^{d}$. However, because of the progressive stiffness degradation from ${ }^{n} \mathbf{E}^{d}$ to ${ }^{n+1} \mathbf{E}^{d}$, the trial stress reduces by a factor of $\left(1-{ }^{n+1} D\right) /\left(1-{ }^{n} D\right)$. The total stress ${ }^{n+1} \boldsymbol{\sigma}$ is therefore calculated by correcting the damaged trial stress for the plastic deformation increment ${ }^{n+1} \Delta \boldsymbol{\varepsilon}^{p}$. In this paper, when both plasticity and damage processes are active, the damaged trial stress 
and plastic correction are both determined implicitly by solving a simultaneous system of nonlinear equations in terms of the plastic multiplier ${ }^{n+1} \Delta \lambda$ and the damage increment ${ }^{n+1} \Delta D$.

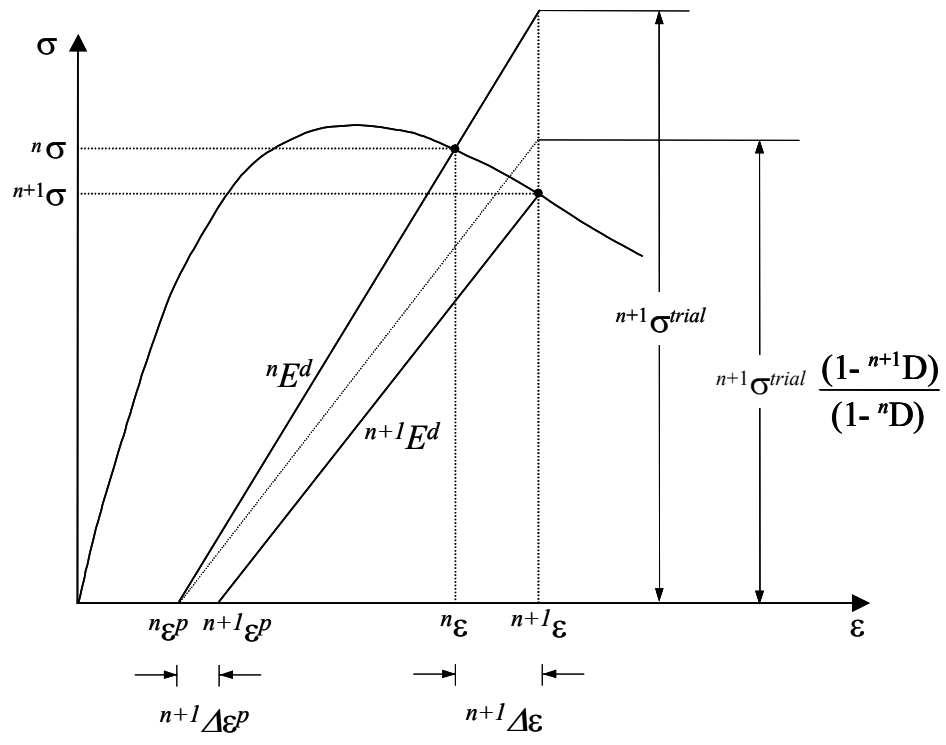

Figure 2. Schematic representation of inelastic damage process

\section{PERFORMANCE OF THE MODEL}

For the purpose of illustration, the coupled elastoplastic damage model was implemented in the finite element analysis code ADINA. The performance of the constitutive formulation model was evaluated for four different load scenarios including uniaxial tension, uniaxial compression, simple shear and confined compression. The results will be discussed in the subsequent section. 
There are twelve material parameters needed to calibrate the elastic, plastic and damage behavior of the geomaterial model. Representative values for sandstone and normal strength concrete are summarized in Table 1.

(a) The undamaged elastic state of the isotropic material is specified by the elastic modulus $E^{\circ}=21500 \mathrm{MPa}$ and Poisson's ratio $v=0.192$.

(b) The plastic properties involve the initial and maximum values of the friction and cohesion parameters of the Drucker-Prager yield function: $\alpha_{0}=\alpha_{m}=0.23$, $k_{0}=6.27 \mathrm{MPa}$ and $k_{m}=8.16 \mathrm{MPa}$. Hereby the parameter $b_{2}=5000$ is used for specifying the exponential hardening rule of the cohesion parameter $k$ in (16) and (17), while no hardening is considered for the friction parameter $\alpha$. To control dilatancy, a non-associated flow rule is used where the dilatancy parameter is $\beta=0.115$.

(c) The plastic damage parameters in (31) which define the level of plastic volumetric work in the thermodynamic force differ in tension from compression, and are set to $c_{t}=1.0$ and $c_{c}=0.1$, respectively. Finally, the energy capacity in the damage resistance function is defined by the exponent $p=0.01$ in (34) which specifies the ratio of the modulus of resilience to the modulus of toughness. Note that the modulus of resilience $r_{\mathrm{o}}$ in the damage resistance function is not an independent material parameter. It corresponds to the strain energy content at peak stress of the uniaxial tension test, which coincides with the yield point in 
tension. Hence it can be determined from the friction and cohesion parameters of the Drucker-Prager yield function.

Calibration of the geomaterial model can be performed through a series of uniaxial tension and compression as well as triaxial compression tests. The modulus of elasticity $E^{\circ}$ and Poisson's ratio $v$ can be determined from the uniaxial compression test, while strength data from uniaxial and triaxial compression tests can be used to determine the friction and cohesion parameters. Note, there are only two strength parameters which may be used to fit either the strength in triaxial compression or in tension-compression. For more comprehensive descriptions the current plasticity model would have to be extended along the line of the 3-parameter model of Menetrey \& Willam [1995] which also accounts for the effect of the third invariant which is quite pronounced in concrete materials. Finally the damage properties of the material need to be determined from a number of cyclic tests in tension and compression.

Table 1. Material Parameters

\begin{tabular}{|c|c|c|c|c|c|c|c|c|c|c|c|}
\hline \multicolumn{2}{|c|}{ Elasticity } & \multicolumn{9}{|c|}{ Plasticity } & \multicolumn{3}{c|}{ Damage } \\
\hline $\begin{array}{c}E^{0} \\
(\mathrm{MPa})\end{array}$ & $v$ & $\alpha_{0}$ & $\alpha_{m}$ & $b_{1}$ & $\begin{array}{c}\kappa_{0} \\
(\mathrm{MPa})\end{array}$ & $\begin{array}{c}\kappa_{m} \\
(\mathrm{MPa})\end{array}$ & $b_{2}$ & $\beta$ & $c_{t}$ & $c_{c}$ & $p$ \\
\hline 21500 & 0.192 & 0.23 & 0.23 & 0 & 6.27 & 8.16 & 5000 & 0.115 & 1.0 & 0.1 & 0.01 \\
\hline
\end{tabular}

\section{RESPONSE PREDICTIONS}

In what follows the triaxial material behavior is analyzed for different load histories under mixed displacement-traction control (full displacement control in the case of simple shear). To this end the response of single hexahedral element is examined under 
uniform load conditions which involve partial unload-reload histories to illustrate the degradation of stiffness due to progressive damage. The main response is plotted in terms of the axial stress-strain components in tension and compression and the in-plane shear components in the case of the simple shear experiment.

The axial response behavior of the geomaterial model under uniaxial tension is shown in Figure 3. The response depicts a linear elastic region which is followed by a sharp deterioration of strength due to activation of the damage process. Observe the significant degradation of the elastic stiffness due to tensile damage which increases with progressive softening.

Figure 4 illustrates the performance of the geomaterial model under uniaxial compression. Three distinctive regions can be observed. Initially the material behaves linearly elastically until initial yield is reached. Thereafter the material shows plastichardening which is accompanied by plastic volume expansion. When the plastic dilatation reaches a critical limit defined by the damage resistance of the material, the damage process is mobilized resulting in large deterioration of strength down to a residual strength level as well as in progressive damage of the elastic stiffness in the softening regime.

Figure 5 illustrates the performance of the geomaterial model under simple shear. In analogy to uniaxial compression the linearly elastic shear response id followed by plastichardening which is accompanied by plastic volume expansion. When the plastic dilatation reaches a critical limit defined by the damage resistance, the damage process is 
mobilized. This results in a significant deterioration of strength which is accompanied by progressive damage of the elastic stiffness in the softening regime.

In order to study the performance of the geomaterial model under confined conditions, two triaxial tests were performed at confining stresses of $p=-4 \mathrm{MPa}$ and $p=-8 \mathrm{MPa}$. The results are compared with that of uniaxial compression in Figure 6. They show that the geomaterial model exhibits a large increase of strength with increasing confinement.

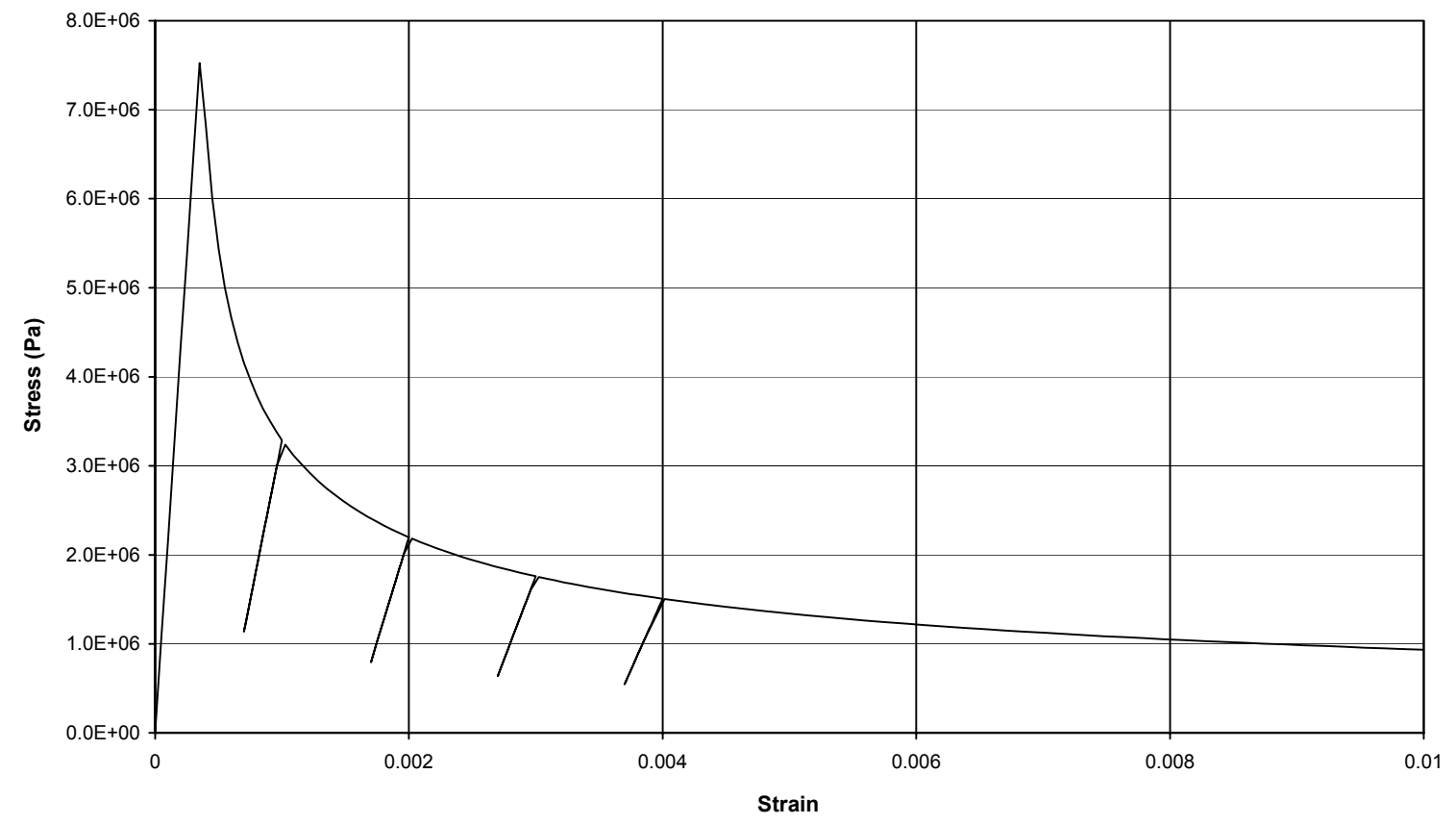

Figure 3. Uniaxial tension test 


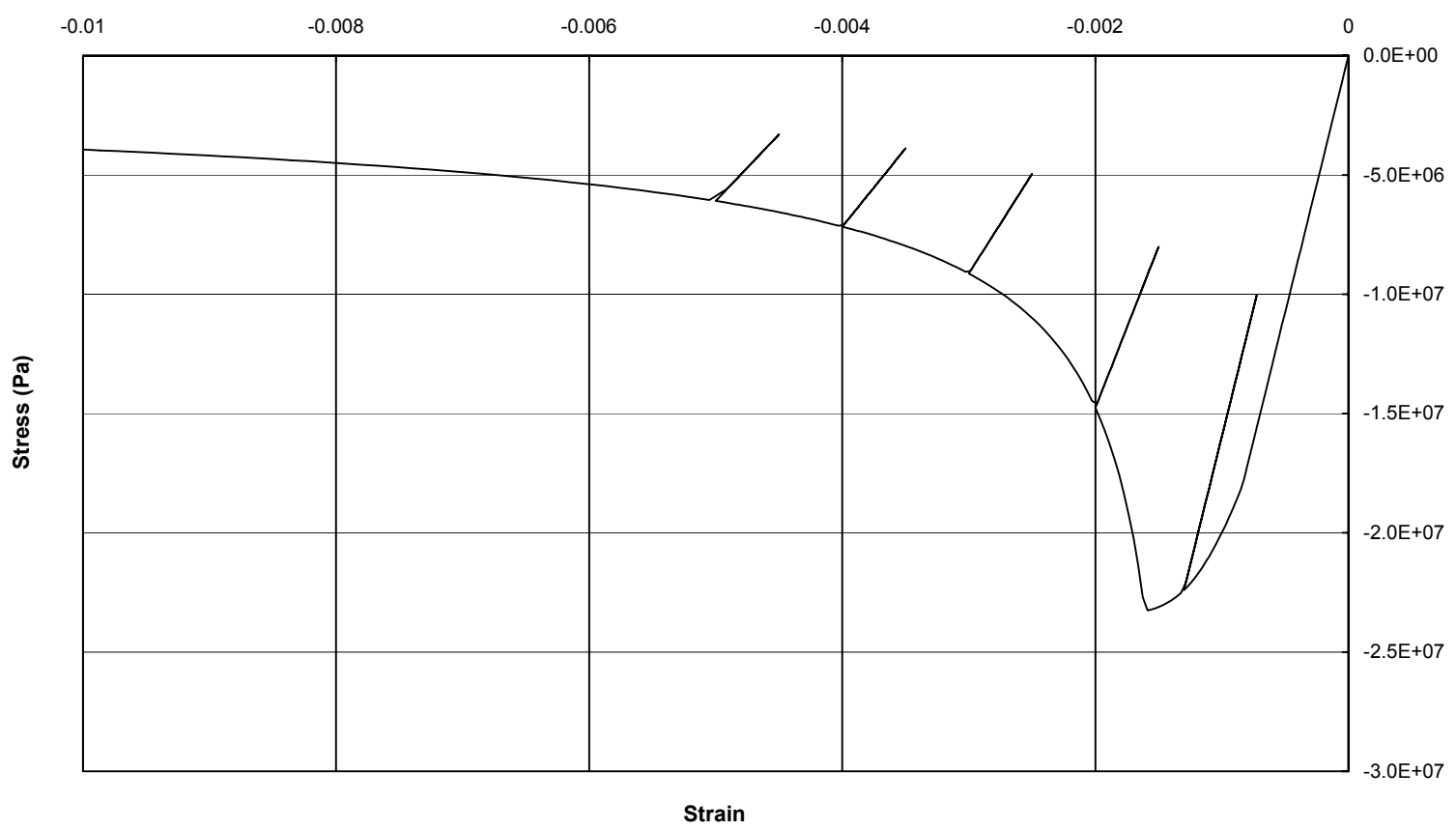

Figure 4. Uniaxial compression test

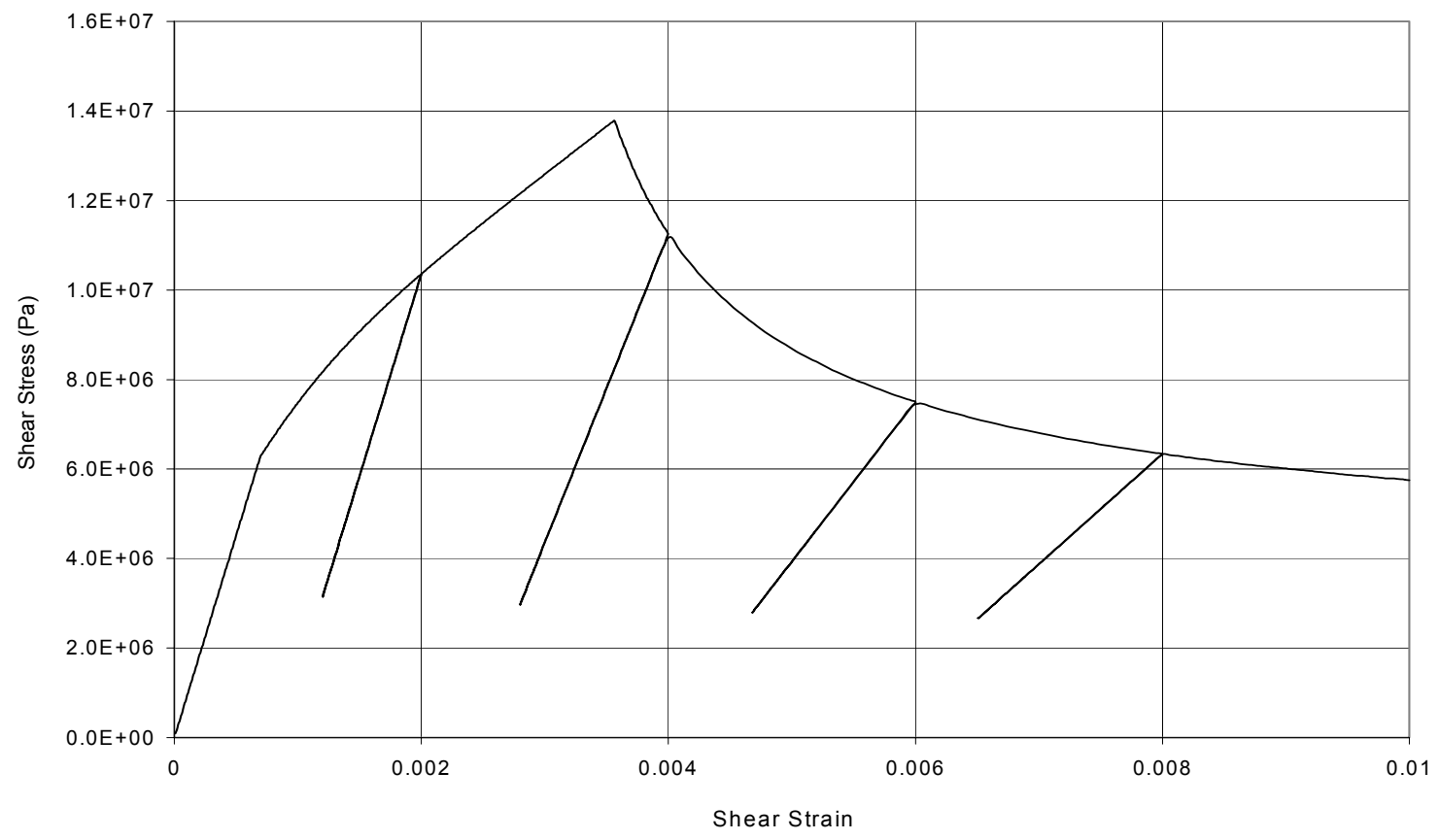

Figure 5. Simple shear test 


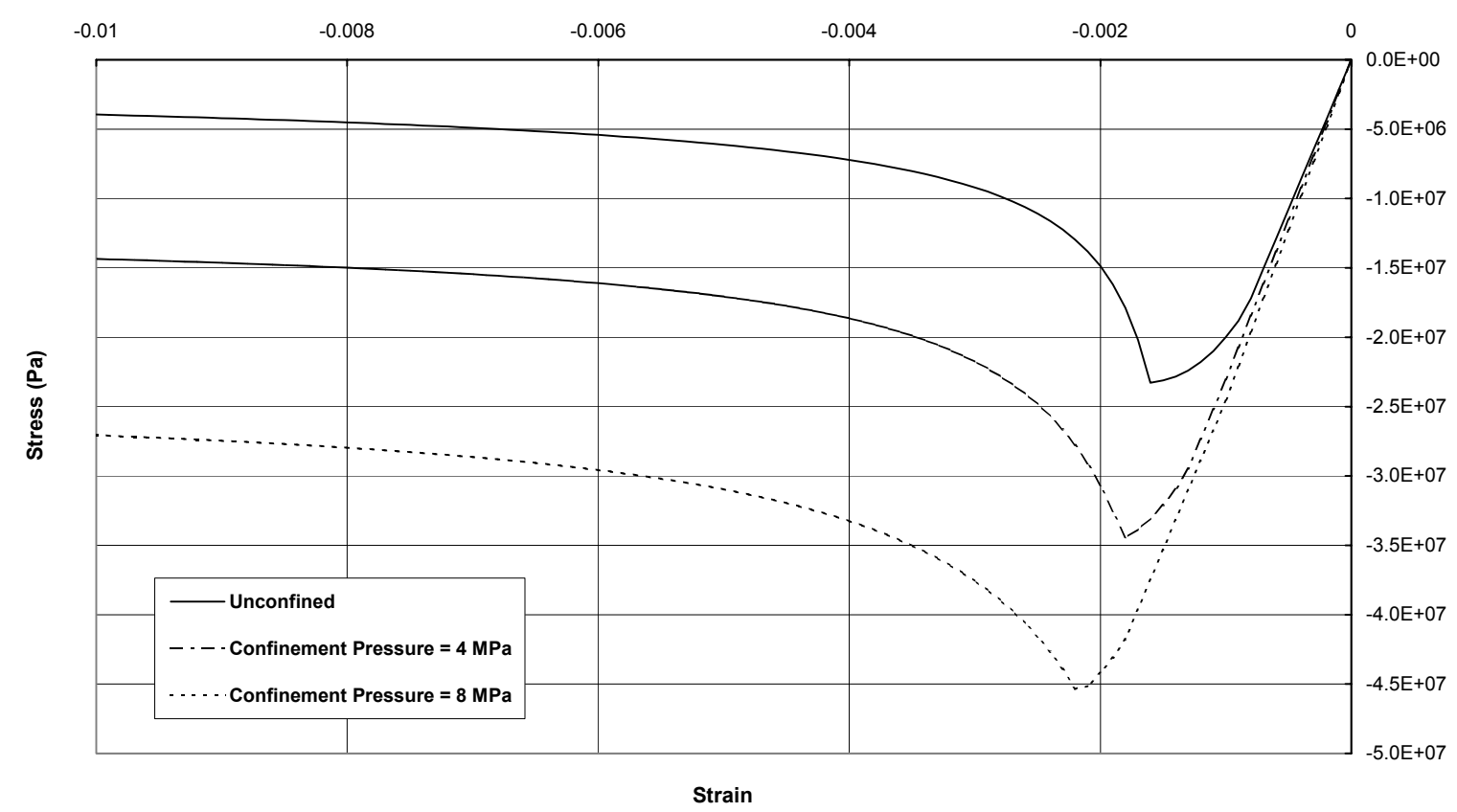

Figure 6. Confined compression test

\section{FAILURE PREDICTIONS}

The issue of well-posedness of the related initial boundary value problem is closely connected to the positive behavior of the tangential material properties and the associated localization tensor. In fact, positivity of the symmetrized material operator provides a sufficient condition for material stability in the sense of positive second order work density, $d^{2} W=\dot{\boldsymbol{\varepsilon}}: \mathbf{E}^{\text {epd }}: \dot{\boldsymbol{\varepsilon}}>0$. At the other hand, positivity of the non-symmetric material operator provides a sufficient condition for uniqueness in the sense of $\operatorname{det}\left(\mathbf{E}^{e p d}\right)>0$. Considering discontinuous bifurcation in the form of localization it is well understood that non-symmetric localization operators $\mathbf{Q}^{\text {epd }}=\mathbf{N} \cdot \mathbf{E}^{\text {epd }} \cdot \mathbf{N}$ often exhibit a singularity in the ascending part of the stress-strain relationship when the tangential material stiffness is still positive, see Rudnicki and Rice [1975]. In contrast, 
symmetric localization operators exhibit a singularity only when the underlying tangent material law turns non-positive.

Consequently, the different material failure diagnostics adhere to the following hierarchy:

(1) loss of stability of the symmetrized material operator, when $\lambda_{\min }\left(\mathbf{E}_{\text {sym }}^{\text {epd }}\right)=0$

(2) loss of ellipticity of the non-symmetric localization tensor, when $\lambda_{\min }\left(\mathbf{Q}^{\text {epd }}\right)=0$

(3) loss of uniqueness of the non-symmetric material operator, when $\lambda_{\min }\left(\mathbf{E}^{\text {epd }}\right)=0$

In what follows, we investigate the different failure diagnostics for the load histories examined before.

Figure 7 illustrates the variation of the lowest eigenvalue of the symmetric and nonsymmetric tangential material operators under uniaxial tension. The figure clearly indicates the positive definiteness in the ascending portion of the tensile response and the drastic sign change of eigenvalues in the softening regime. Thereby it is understood that the minimum eigenvalue of the tangential material law is a manifestation of the second order work density, either stored or released, depending on its sign. Thereby the corresponding eigenvector would indicate the strain rate associated with the failure mode when $\lambda_{\min }\left(\mathbf{E}^{e p d}\right)<0$. We note the lower bound of the symmetrized tangent operator when compared to the non-symmetric operator. This property is a result of the so-called Bromwich bounds of linear algebra. Furthermore, we observe the strong influence of the softening slope on the negative value of the energy release rate which decreases significantly near the tail of the experiment. 
Figure 8 illustrates the corresponding localization result when the tensile stress reaches the peak value in uniaxial tension. At this stage the normalized determinant of the localization tensor, $\operatorname{det}\left(\mathbf{E}^{e p d}\right) / \operatorname{det}\left(\mathbf{E}^{e}\right)<0$, reaches a minimum value at $\theta=0,180$ degrees. These two angles define a single normal vector to the failure plane with regard to the major principal axis indicating mode I cracking in uniaxial tension. Note that the large negative value of the normalized determinant exceeds the corresponding positive value of the elastic acoustic tensor by a factor of 2.5. This indicates that both, the associative as well as the non- associative localization tensors indicate discontinuous bifurcation, Rudnicki [2002], and hence formation of a discontinuity in the solution domain from a material zone subject to loading to a material zone subject to unloading.

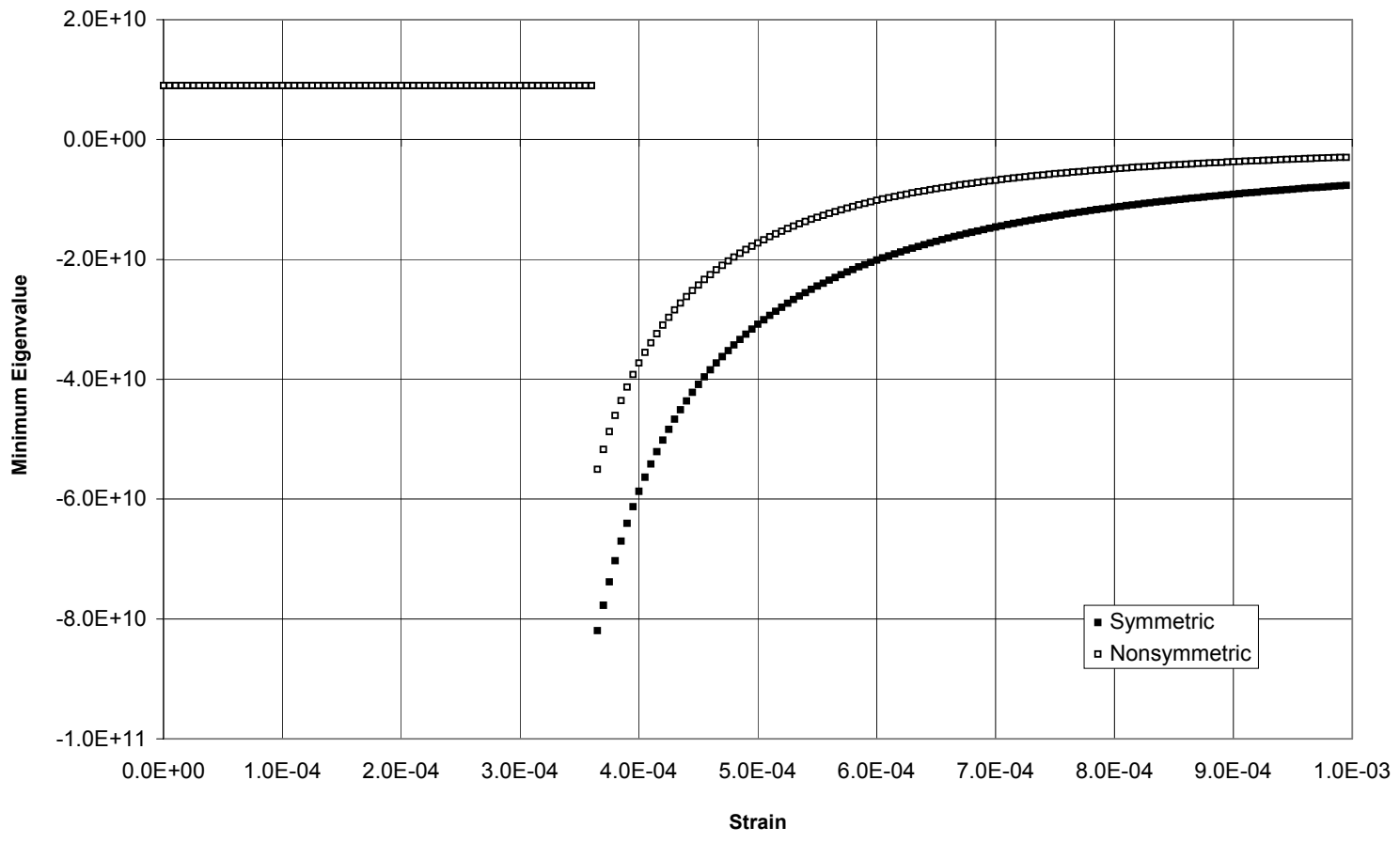

Figure 7. Lowest eigenvalue vs. strain for uniaxial tension test 


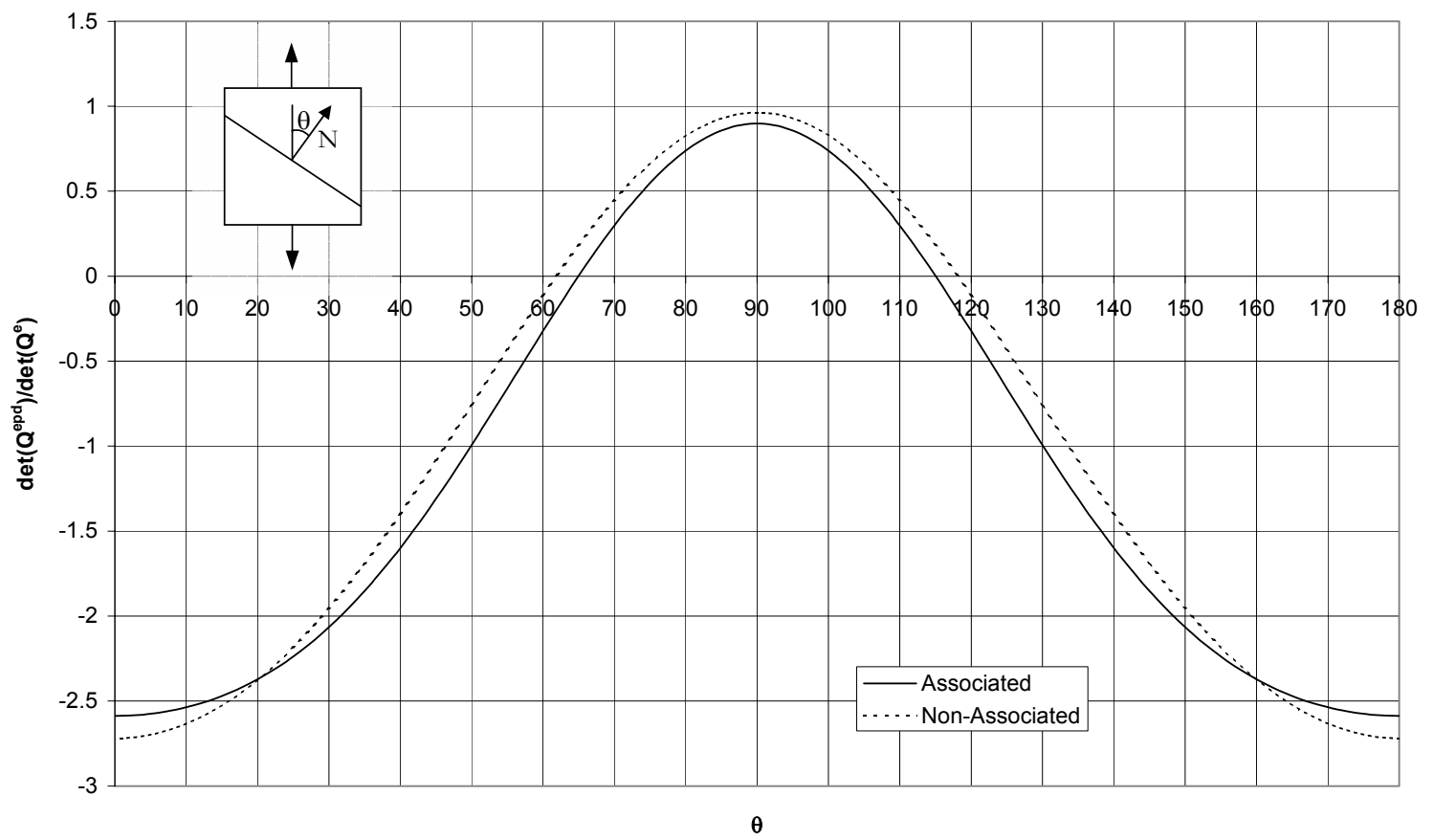

Figure 8. Non-symmetric localization analysis of uniaxial tension test comparing associative with non-associative flow rules

Figures 9 and 10 illustrate the analogous features of the geomaterial model in uniaxial compression. In this case we note the loss of positive behavior of the symmetrized material stiffness in the hardening regime and again the drastic change from energy storage to energy release when the response reaches the peak stress level and the onset of softening. A snap shot of the localization properties at that instant is shown in Figure 10. It indicates discontinuous bifurcation when the associative localization operator is considered, but not for the non- associative one. In other terms, loss of strong ellipticity is indicated by the negative value of the associative localization tensor, while loss of ellipticity in the form of zero values of the determinant is not being reached by the nonassociative localization tensor. In the former case the normal vectors of the localized slip planes tend to form at two distinct angles $\theta=\sim 40$ and $\sim 140$ degrees with regard to the major principal axis. Thereby the associative localization diagnostics provide a lower 
bound of the non- associative localization properties which remain positive. In other terms, the cylindrical stress states of uniaxial and triaxial compression prevent localization in spite of the negative material properties due to softening and nonassociated plastic flow, see also Kang and Willam [1999] for this surprising observation.

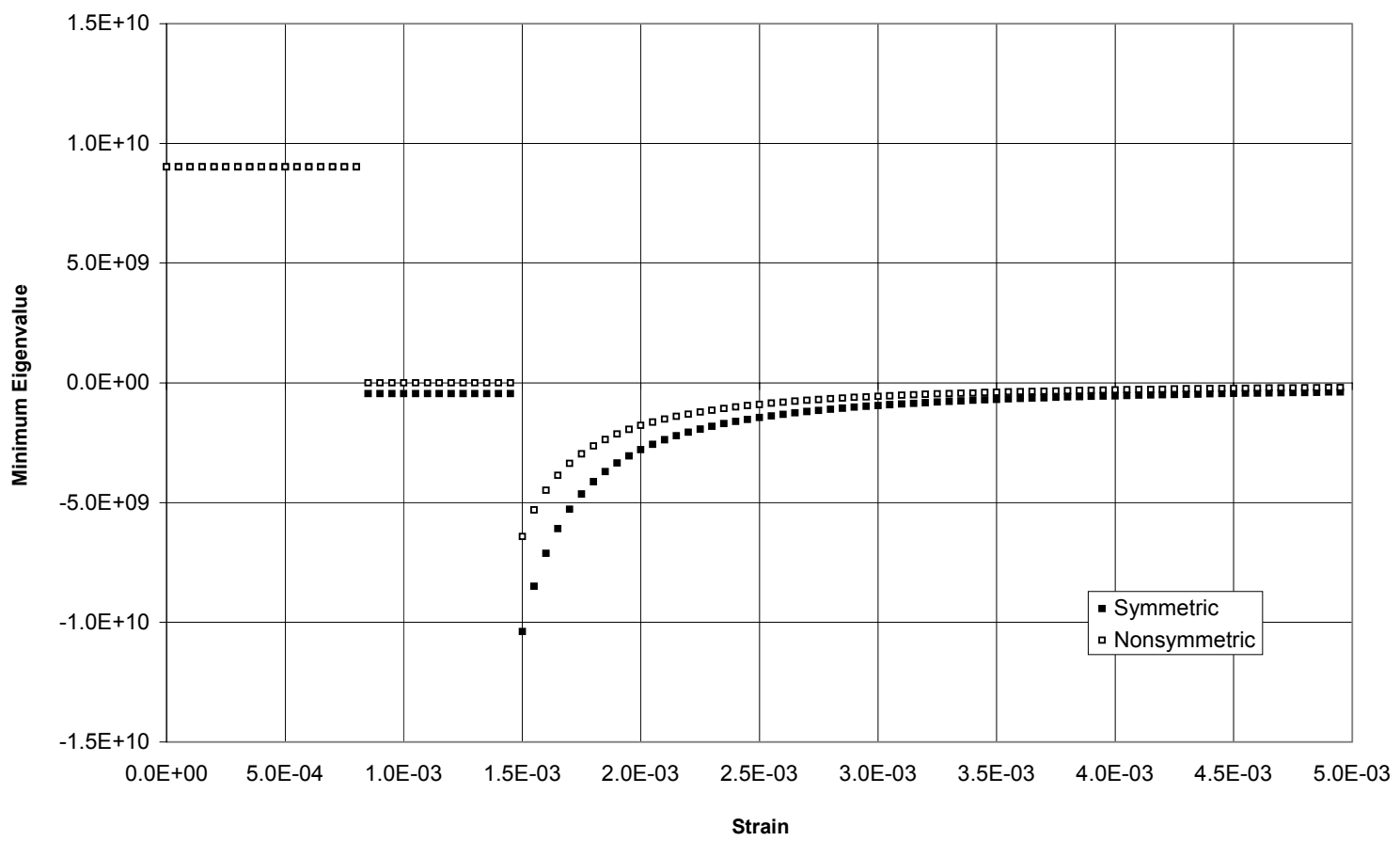

Figure 9. Lowest eigenvalue vs. strain for uniaxial compression test 


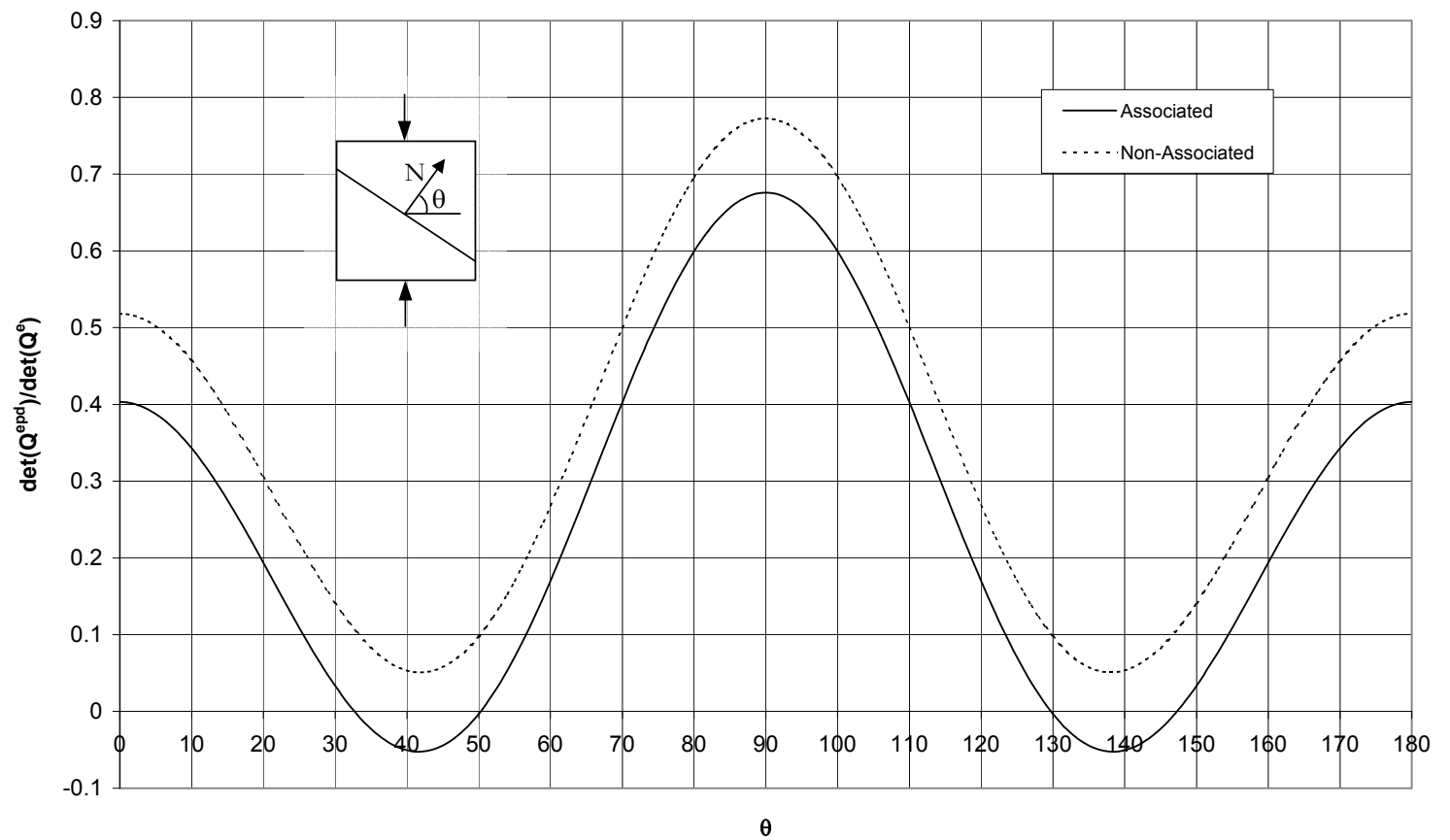

\section{Figure 10. Non-symmetric localization analysis of uniaxial Compression test comparing associative with non-associative flow rules}

Figures 11 and 12 illustrate the analogous features of the geomaterial model in simple shear. In this case we note again the loss of positive behavior of the symmetrized material stiffness in the hardening regime and the drastic change from energy storage to energy release when the response reaches the peak stress level and the onset of softening. A snap shot of the localization properties at that instant is shown in Figure 12 which indicates discontinuous bifurcation when both the associative as well as the nonassociative localization operators are considered. In this case localization takes place at two distinct planes the normals of which are oriented at the angles $\theta=\sim 39$ and $\sim 141$ degrees with regard to the major principal axis. Thereby the directional properties of the localized failure planes are independent of the flow rule, while the onset of localization and the transition from continuous to discontinuous bifurcation differ strongly between the associative and the non-associative failure diagnostics. 


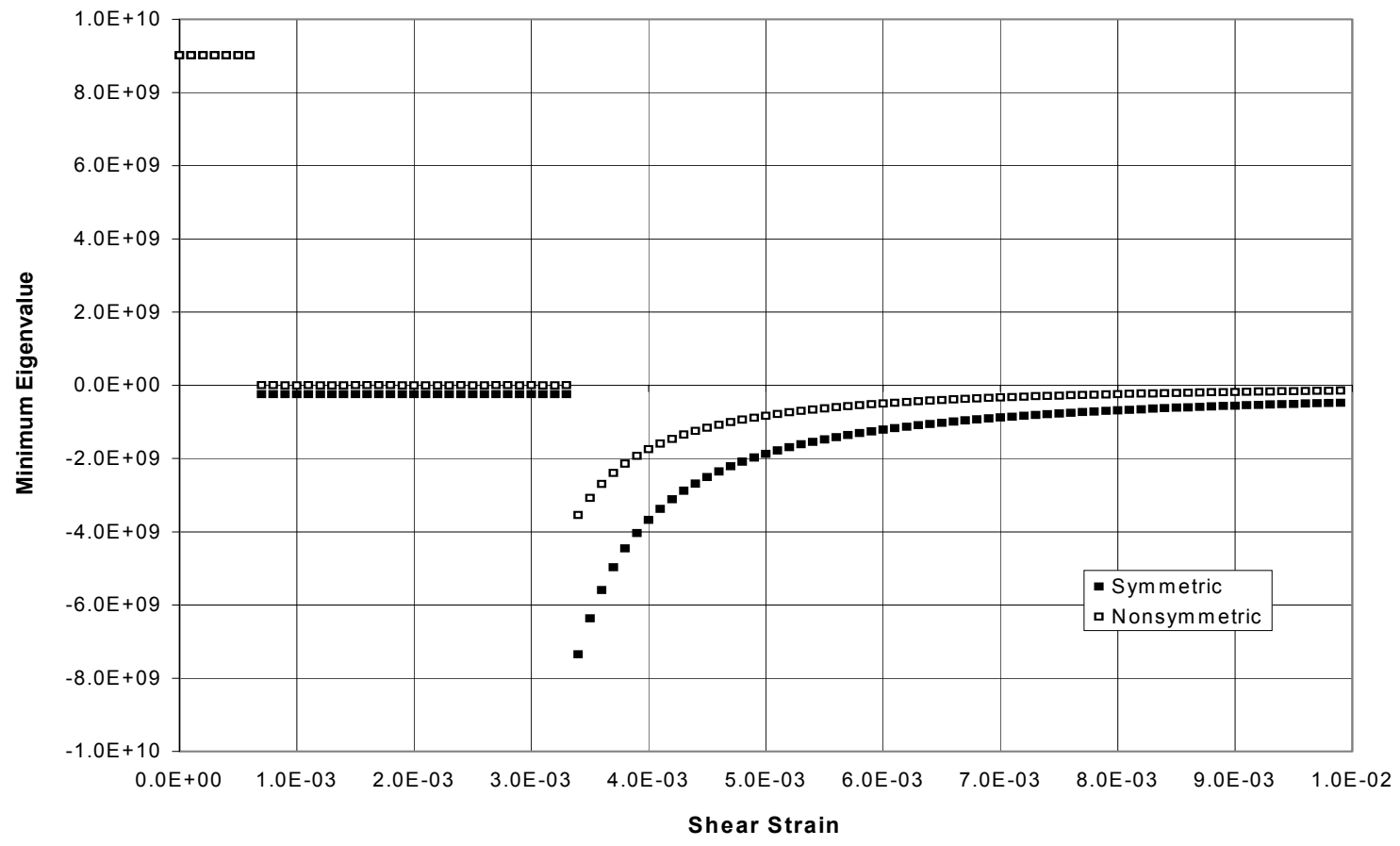

Figure 11. Lowest eigenvalue vs. shear strain for simple shear test

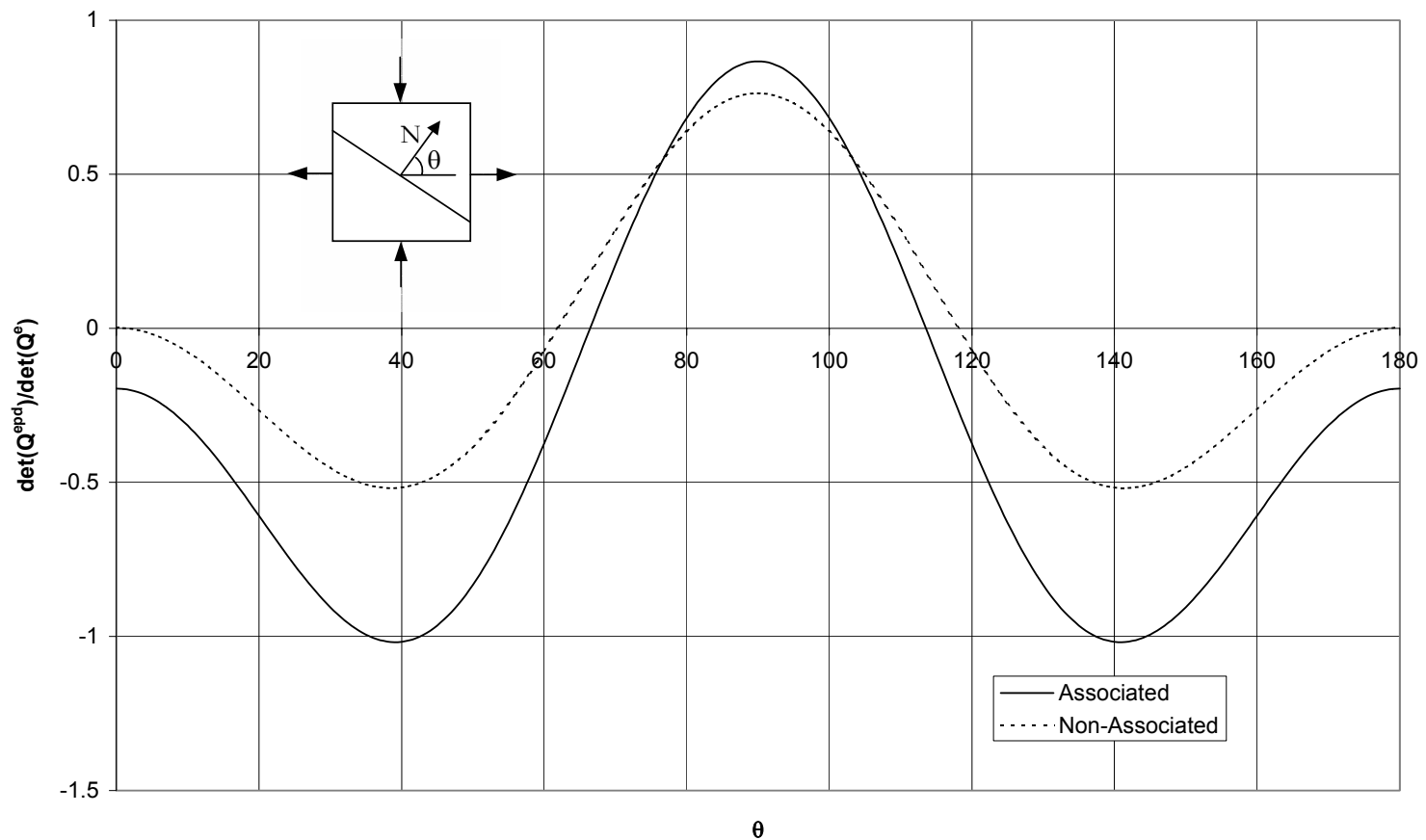

Figure 12. Non-symmetric localization analysis of uniaxial simple shear test comparing associative with non-associative flow rules

\section{SUMMARY AND CONCLUSION}


A triaxial model was presented for geomaterials based on two interacting dissipation mechanisms, one for elastoplastic behavior and one for elastic damage. Coupling of the two dissipation mechanisms was introduced and controlled by volumetric expansion. The isotropic geomaterial model involves twelve parameters, two define the initial elastic stiffness, seven hardening and softening parameters describe non-associated plastic flow, and three characterize the evolution of scalar damage.

Four load histories were examined in the example problems to illustrate the performance of the geomaterial model. The failure diagnostics revealed a number of important issues related to the loss of positive tangent properties and the formation of discontinuous failure modes due to localization beyond which uniform deformations cease to exist.

Contrary to the destabilizing effect of associative vs. non-associative flow formulations, the mode of localized failure showed little effect of the flow rule. Opposite to the localization tendencies during softening in uniaxial tension and simple shear, the localization properties remained remarkably positive in uniaxial compression in spite of softening and non-associative plastic flow.

\section{ACKNOWLEDGEMENT}

The third author wishes to acknowledge partial support of this research by the National Science Foundation under grant CMS-0084598.

\section{REFERENCES}

ADINA R\&D, 2001, Theory and Modeling Guide, Volume I: ADINA, Report ARD 017, Watertown, MA. 
Borja, R.I., Tamagnini, C., (1998), “Cam-ClayPlasticity, Part III: Extension of the Infinitesimal Model to Include Finite Strains", Computer Methods in Applied Mechanics and Engineering, Vol. 155, pp.73-95.

Carol, I., Rizzi, E. and Willam, K., (2001), "On the Formulation of Anisotropic Degradation. I. Theory based on a Pseudo-Logarithmic Damage Tensor", Intl. J. of Solids and Structures, Vol. 38/4, pp. 491-518.

Carol, I., Rizzi, E. and Willam, K., (2001), "On the Formulation of Anisotropic Degradation. II. Generalized Pseudo-Rankine Model for Tensile Damage", Intl. J. of Solids and Structures, Vol. 38/4, pp. 519-546.

Dafalias, Y.F., (1986), “Bounding Surface Plasticity. Part I: Mathematical Foundation and Hypoplasticity", Journal of Engineering Mechanics, ASCE, Vol. 112, pp. 966987.

Darve, F., (1991), “Incrementally Nonlinear Constitutive Relationship" in Geomaterials: Constitutive Equation and Modelling, Darve (ed.) Elsevier Amsterdam, pp. 213-237.

Desai, C., (2001), "Mechanics of Materials and Interfaces: The Disturbed State Concept", CRC Press, Boca Baton, Florida.

Dragon, A. and Mroz, Z., (1979), “A Continuum Model for Plastic-Brittle Behaviour of Rock and Concrete”, Intl. J. Engrg. Sci, Vol. 17, pp. 121-137.

Hansen, E., Willam, K. and Carol, I., (2001), “A Two-Surface Anisotropic Damage/Plasticity Model for Plain Concrete", Proc. 4th Int. Conf. Fracture Mechanics 
of Concrete Materials, Framcos-4, Paris, May 28-31, 2001, R. de Borst, J. Mazars, G. Pijaudier-Cabot and J. van Mier (eds.), A.A. Balkema, Rotterdam, 2001, pp. 549-556.

Kang, H. and Willam, K., (1999), "Localization Characteristics of a Triaxial Concrete Model," ASCE-JEM, Vol. 125, pp. 941-950.

Kolymbas, D., (1991), “An Outline of Hypoplasticity”, Archives of Applied Mechanics, Vol. 61, pp. 143-151.

Lee, J. and Fenves G.L. , (1998), "Plastic-Damage Model for Cyclic Loading of Concrete Structures”, Journal of Engineering Mechanics, ASCE, Vol. 124, pp. 892-900.

Mazars, J. and Pijaudier-Cabot, G., (1989), "Continuum DamageTheory-Application to Concrete", Journal of Engineering Mechanics, ASCE, Vol. 115, pp. 345-365.

Menetrey, Ph. and Willam, K., (1995), “A Triaxial Failure Criterion for Concrete and its Generalization,” ACI Structures Journal, Vol. 92, pp. 311-318.

Nawrocki, P.A., and Mroz, Z., (1999), “A constitutive model for Rock Accounting for viscosity and yield stress degradation," Computers and Geotechnics, Vol. 25, pp. 247280.

Nova, R., (1992), "Mathematical Modelling of Natural and Engineered Geomaterials", European Journal of Mechanics/ A Solids, pp. 135-154.

Rudnicki, J.W. and Rice, J.R., (1975), "Conditions for the Localization of Deformation in Pressure Sensitive Dilatant Materials", Journal of the Mechnics and Physics of Solids, Vol. 23, pp. 371-394. 
Rudnicki, J.W. (2002), “Condition for Compaction and Shear Bands in a Transversely Isotropic Material”, Intl. J. of Solids and Structures, Vol. 39, pp. 3741-3756.

Shao, J.F., Chiarelli, A.S., Hoteit, N., (1998), "Modeling of Coupled Elastoplastic Damage in Rock Materials," Intl. J. of Rock Mech. \& Min. Sci., Vol. 35, Nos. 4-5, Paper No. 115.

Simo, J.C. and Hughes, T.J.R., (1998), Computational Inelasticity, Springer-Verlag New York, Inc.

Valanis K.C. and Peters, J.F., (1996), "Ill-Posedness of the Initial and Boundary Value Problems in Non-Associative Plasticity", Acta Mechnaica, Vol. 114, pp. 1-15.

Willam, K.J., (2002), “Constitutive Models for Engineering Materials," Encyclopedia of Physical Science \& Technology, 3rd Edition, Volume 3, Academic Press, pp. 603-633. http://civil.colorado.edu/ willam/mat101.pdf

Yazdani, S. and Schreyer, H.L., (1988), “An Anisotropic Damage Model with Dilatation for Concrete", Mechanics of Materials, Vol. 7, pp. 231-244.

\section{NOTATION}

The following symbols are used in this paper:

$\begin{array}{ll}c_{c}, c_{t} & \text { plastic participation factors in damage force } \\ D & \text { scalar damage parameter } \\ \mathbf{E}^{0} & \text { initial elasticity tensor }\end{array}$




\begin{tabular}{|c|c|}
\hline $\mathbf{E}^{d}$ & secant tensor of elastic damage \\
\hline $\mathbf{E}^{e d}$ & tangential elastic damage operator \\
\hline $\mathbf{E}^{e p}$ & tangential elastoplastic operator \\
\hline $\mathbf{E}^{e p d}$ & tangential elastoplastic damage operator \\
\hline$\dot{\mathbf{e}}^{p}$ & deviatoric plastic strain rate \\
\hline $\bar{e}^{p}$ & equivalent deviatoric plastic strain \\
\hline$f_{c}, f_{t}$ & uniaxial compressive and tensile strengths \\
\hline$f^{d}$ & damage function \\
\hline$f^{p}$ & plastic loading function \\
\hline$g^{d}$ & damage potential function \\
\hline$g_{f}$ & volumetric fracture energy release per unit volume \\
\hline$g^{p}$ & plastic potential function \\
\hline $\mathbf{I}$ & $2^{\text {nd }}$ order identity tensor \\
\hline$I_{1}$ & first invariant of the nominal stress tensor \\
\hline$J_{2}$ & second invariant of the nominal deviatoric stress tensor \\
\hline$K$ & thermodynamic conjugate force for plasticity \\
\hline$K^{0}$ & undamaged bulk modulus \\
\hline$k$ & Drucker-Prager cohesion parameter \\
\hline$k_{0}, k_{m}$ & initial and maximum values of $k$ \\
\hline$p$ & ratio between modulus of resilience and modulus of toughness \\
\hline$r$ & resistance function \\
\hline
\end{tabular}




\begin{tabular}{|c|c|}
\hline$r_{\mathrm{o}}$ & modulus of resilience \\
\hline $\mathbf{s}$ & deviatoric strain tensor \\
\hline$Y$ & thermodynamic conjugate force for damage \\
\hline$Y_{v}$ & volumetric thermodynamic conjugate force for damage \\
\hline$\alpha$ & Drucker-Prager friction parameter \\
\hline$\alpha_{0}, \alpha_{m}$ & initial and maximum values of $\alpha$ \\
\hline$\beta$ & dilatation parameter \\
\hline $\boldsymbol{\varepsilon}^{e}$ & elastic strain tensor \\
\hline $\boldsymbol{\varepsilon}^{p}$ & plastic strain tensor \\
\hline$\dot{\varepsilon}_{m}^{p}$ & mean plastic strain rate \\
\hline$\varepsilon_{v}^{e}$ & volumetric elastic strain \\
\hline$\kappa$ & internal variable of plasticity \\
\hline$\dot{\lambda}$ & plastic multiplier \\
\hline$\dot{\mu}$ & damage multiplier \\
\hline$\sigma$ & nominal stress tensor \\
\hline$\sigma_{m}$ & mean nominal stress \\
\hline$\psi$ & Helmholtz free energy \\
\hline$\psi^{e}$ & elastic component of Helmholtz free energy \\
\hline$\psi^{p}$ & plastic component of Helmholtz free energy \\
\hline
\end{tabular}

